\title{
MiR21 sensitized B-lymphoma cells to $A B T-199$ via ICOS/ICOSL-mediated interaction of Treg cells with endothelial cells
}

Zhong Zheng ${ }^{1 \dagger}$, Peng-Peng X $u^{1,2+}$, Li Wang ${ }^{1,2+}$, Hui-Jin Zhao ${ }^{1 \dagger}$, Xiang-Qin Weng ${ }^{1}$, Hui-Juan Zhong ${ }^{1}$, Bin Qu ${ }^{3}$, Jie Xiong ${ }^{1}$, Yan Zhao ${ }^{1}$, Xue-Feng Wang ${ }^{3}$, Anne Janin ${ }^{2,4}$ and Wei-Li Zhao ${ }^{1,2^{*}}$

\begin{abstract}
Background: MicroRNAs (miRs) are involved in tumor progression by regulating tumor cells and tumor microenvironment. MiR21 is overexpressed in diffuse large B-cell lymphoma (DLBCL) and its biological impact on tumor microenvironment remains unclear.

Methods: MiR21 was assessed by quantitative RT-PCR in patients with newly diagnosed DLBCL. The mechanism of action of miR21 on lymphoma progression and tumor angiogenesis was examined in vitro in B-lymphoma cell lines and in vivo in a murine xenograft model.

Results: Serum miR21 was significantly elevated in patients and associated with advanced disease stage, International Prognostic Index indicating intermediate-high and high-risk, and increased tumor angiogenesis. When co-cultured with immune cells and endothelial cells, miR21-overexpressing B-lymphoma cells were resistant to chemotherapeutic agents, but sensitive to Bcl-2 inhibitor ABT-199, irrespective of Bcl-2 expression on lymphoma cells. In both co-culture systems of $\mathrm{BCl}-2^{\text {positive }}$ and $\mathrm{BCl}-2^{\text {negative }} \mathrm{B}$-lymphoma cells, miR21 induced inducible co-stimulator (ICOS) expression on regulatory $T$ (Treg) cells. Through crosstalking with Treg cells by ICOS ligand (ICOSL), endothelial cells were activated, resulting in stimulation of $\mathrm{BCl}-2$ expression and vessel formation. ABT-199 directly targeted Bcl-2 on endothelial cells, induced endothelial cell apoptosis and inhibited tumor angiogenesis. In a murine xenograft model established with subcutaneous injection of B-lymphoma cells, ABT-199 particularly retarded the growth of miR21-overexpressing tumors, consistent with the induction of endothelial cell apoptosis and inhibition of tumor angiogenesis.
\end{abstract}

Conclusions: As a serum oncogenic biomarker of B-cell lymphoma, miR21 indicated B-lymphoma cell sensitivity to ABT-199 via ICOS/ICOSL-mediated interaction of Treg cells with endothelial cells.

Keywords: MicroRNA21, B-cell lymphoma, ABT-199, Tumor microenvironment, Regulatory T cells, Endothelial cells

\footnotetext{
*Correspondence: zhao.weili@yahoo.com

${ }^{\dagger}$ Equal contributors

${ }^{1}$ State Key Laboratory of Medical Genomics, Shanghai Institute of

Hematology, Shanghai Rui Jin Hospital, Shanghai Jiao Tong University School

of Medicine, 197 Rui Jin Er Road, Shanghai 200025, China

${ }^{2}$ Pôle de Recherches Sino-Français en Science du Vivant et Génomique,

Laboratory of Molecular Pathology, Shanghai, China

Full list of author information is available at the end of the article
} 


\section{Background}

Diffuse large B-cell lymphoma (DLBCL) represents the most common neoplastic disorder of B-lymphocytes. Although addition of anti-CD20 antibody Rituximab to conventional chemotherapy has significantly improved the clinical outcome of the patients, due to disease heterogeneity, relapse and refractory to immunochemotherapy remains of great concern [1]. As a newly developed biotherapeutic agent, ABT-199 is a specific Bcl-2 inhibitor targeting the $\mathrm{BH} 3$-binding domains of $\mathrm{Bcl}-2$ and has demonstrated encouraging preclinical results in treating B-cell lymphoma [2, 3]. However, the underlying mechanism of ABT-199 in treating DLBCL warrants further investigation.

Besides genetic abnormalities of malignant cells themselves, the aberrant status of tumor microenvironment plays a pivotal role on disease progression [4]. As the main components of microenvironment, tumor vessels prevent the attack of chemotherapy on tumor cells [5]. Moreover, tumor angiogenesis mediated by immune cells is involved in the development of drug resistance in lymphoma [6, 7]. Direct interaction of immunosuppressive regulatory $\mathrm{T}$ (Treg) cells with vascular endothelial cells contributes to the regulation of anti-lymphoma responses $[8,9]$. Inducible co-stimulator (ICOS) and its ligand ICOSL function as an important crosstalk between immune cells and endothelial cells [10]. Since it has recently been reported that the cytotoxic effect of ABT-199 on tumor cells rely on survival signals from tumor microenvironment [11], microenvironmentrelated biomarkers may become potential predictors of ABT-199 efficacy on B-cell lymphoma.

MicroRNAs (miRs), a class of 19- to 23-nucleotide non-coding RNA molecules, regulate gene expression by targeting mRNA at the 3'-untranslated region [12]. In addition to their oncogenic action on tumor cells mediated by oncogenes and/or tumor suppressor genes, miRs are emerging as key regulators of tumor microenvironment $[13,14]$. Among them, miR21 can target Bcl-2 expression by binding to Bcl-2 3'-untranslated region (610-617 bp) and stably expressed in peripheral blood and closely related to disease outcome in B-cell lymphoma as previously reported [15-17]. In the present study, we assessed serum miR21 expression in a large cohort of DLBCL patients and revealed the biological function of miR21 on tumor microenvironment both in vitro and in vivo. Although potentially oncogenic, miR21 enhanced the sensitivity of B-lymphoma cells to ABT199, through an alternative mechanism involving tumor angiogenesis.

\section{Methods}

\section{Patients}

A total of 203 patients with newly diagnosed DLBCL were enrolled in this study. The main clinical characteristics of
Table 1 Clinical characteristics of patients with DLBCL $(n=203)$

\begin{tabular}{|c|c|c|c|}
\hline & High miR21 & Low miR21 & $P$-value \\
\hline \multicolumn{4}{|l|}{ Age } \\
\hline$>60$ years & 35 & 39 & 0.523 \\
\hline$\leq 60$ years & 67 & 62 & \\
\hline \multicolumn{4}{|l|}{ Sex } \\
\hline Female & 39 & 41 & 0.731 \\
\hline Male & 63 & 60 & \\
\hline \multicolumn{4}{|l|}{ Ann Arbor stage } \\
\hline | to || & 50 & 65 & 0.027 \\
\hline III to IV & 52 & 36 & \\
\hline \multicolumn{4}{|l|}{ Extranodal involvement } \\
\hline No & 18 & 17 & \\
\hline Yes & 84 & 84 & 0.882 \\
\hline \multicolumn{4}{|l|}{ Serum LDH level } \\
\hline Normal & 53 & 68 & 0.071 \\
\hline Abnormal & 49 & 33 & \\
\hline \multicolumn{4}{|l|}{ IPI score } \\
\hline Low and intermediate-low risk & 59 & 75 & 0.013 \\
\hline Intermediate-high and high risk & 43 & 26 & \\
\hline
\end{tabular}

$\mathrm{LDH}$ lactate dehydrogenase, IPI International Prognostic Index

the patients were listed in Table 1. Paraffin tumor samples of 50 DLBCL patients were used for immunohistochemistry study. One hundred healthy volunteers were referred as normal control. The study was approved by the Shanghai Rui Jin Hospital Review Board with informed consent obtained from all subjects in accordance with the Declaration of Helsinki.

\section{Cells and reagents}

Human B-lymphoma cell lines SU-DHL-4, SU-DHL-8, human umbilical vein endothelial cell (HUVEC), and murine B-lymphoma cell line A20 were obtained from American Type Culture Collection (Manassas, VA, USA). Cells were cultured in humidified atmosphere of $95 \%$ air and $5 \% \mathrm{CO}_{2}$ at $37{ }^{\circ} \mathrm{C}$. ABT-199 was purchased from Selleck-Biotool (Houston, TX, USA). Anti-Human ICOS functional grade purified antibody was from Affymetrics Ebioscience (San Diego, CA, USA).

\section{Serum and tissue miR21 detection}

Total serum miRNA was extracted using miRNeasy Serum/Plasma Kit (Qiagen, Valencia, CA, USA). MiR21 was measured by real-time quantitative RT-PCR using miScript reverse transcription kit, hsa-miR21 primer and miScript SYBR Green PCR kit (Qiagen). MiR39 was used as endogenous control and DB cells for calibration. Total tissue miRNA was extracted using Trizol agent (Invitrogen, Carlsbad, CA, USA). RNU6 was used as endogenous control and DB cells for calibration. The 
reactions were analyzed on 7500HT Fast Real-time PCR system (Applied Biosystem, Carlsbad, CA, USA). A relative quantification was calculated using the ${ }^{2-\Delta \Delta} C T$ method.

\section{Cell proliferation assay}

Cell proliferation assay was performed as previously described [18].

\section{In vitro co-culture system}

Transwell cell culture chambers $(1 \mu \mathrm{M}$, Millipore Corporation, Billerica, MA, USA) were used for coculture assay. In the co-culture system, lymphoma cells were plated on the upper chamber, with immune cells and HUVEC monolayer on the lower chamber, allowing direct contact of HUVEC with immune cells. Immune cells were mononuclear cells isolated from peripheral blood of healthy volunteers using Ficoll by density gradient centrifugation.

\section{Cell transfection}

SU-DHL-4 and SU-DHL-8 cells were transfected with miR21 mimics (Riobio, Guangzhou, China) or negative control (Riobio) using lipofectamine 2000 (Invitrogen) following the manufacturer's instruction. For the knockdown assay, SU-DHL-4, SU-DHL-8 cells, and HUVEC were transfected with $\mathrm{Bcl}-2$ siRNA or control siRNA (Origene, Rockville, MD, USA) using lipofectamine 2000.

\section{Luciferase report assay}

HEK-293 T cells were transfected with luciferase reporter and miR21 mimics, using Lipofectamine 2000 (Invitrogen) according to the manufacturer's instructions. Protein was collected $24 \mathrm{~h}$ after transfection, using the Passive Lysis Buffer $(30 \mu \mathrm{L}$ per well) provided as part of the Dual-Luciferase Reporter Assay System kit (Promega). Firefly and Renilla luciferase activities were examined by the Dual-Luciferase Reporter Assay System and detected by a Centro XS3 LB960 Luminometer (Berthold).

\section{Lentivirus packaging and transduction}

To overexpress miR21 in A20 cells, purified plasmids pGMLV-miR21 or control vector were transfected into HEK-293 T cells with package vectors using lipofectamine 2000. The supernatant of HEK-293 T cell culture was then condensed to a viral concentration of approximately $3 \times 10^{8}$ transducing units $/ \mathrm{ml}$. The lentiviral particles were incubated with A20 cells for $8 \mathrm{~h}$. The stably transduced cells were selected by green fluorescence protein.

\section{Flow cytometry}

SU-DHL-4 cells were sorted by EasySep ${ }^{\text {Tx }}$ Human CD20+ Cell Isolation Kit, Treg cells by EasySep ${ }^{\mathrm{Ts}}$ Human CD4 + CD127lowCD25+ Regulatory T Cell Isolation Kit (STEMCELL, Vancouver, BC, Canada), and HUVEC by CD31 microbeads kit (Miltenyi Biotec. Shanghai, China). Purity of the sorted populations was greater than 98\%. ICOS expression on Treg cells was assessed using anti-ICOS antibody (Abcam, Cambridge, UK) as the primary antibody and goat anti-mouse IgG H\&L (Abcam) as the secondary antibody. ICOSL expression on HUVEC was assessed using anti-ICOSL antibody (Abcam) as the primary antibody and donkey anti-rabbit IgG H\&L (Abcam) as the secondary antibody. The median fluorescent intensity (MFI) was measured by flow cytometry. Cell cycle and apoptosis analysis were conducted as previously described [19].

\section{Western blot}

Cells were collected and lysed in $200 \mu \mathrm{L}$ lysis buffer (Sigma Aldrich, Shanghai, China). Protein lysates $(20 \mu \mathrm{g})$ were electrophoresed on $10 \%$ sodium dodecylsulfate polyacrylamide gels and transferred to nitrocellulose membranes. Membranes were blocked with 5\% non-fat dried milk and incubated overnight at $4{ }^{\circ} \mathrm{C}$ with appropriate primary antibody, followed by horseradish peroxidase-linked secondary antibody. The immunocomplexes were visualized using chemiluminescence phototope-horseradish peroxidase Kits. Antibodies against Bcl-2, PDCD4, STAT3, p-STAT3 were from Cell Signaling Technology (Danvers, MA, USA). $\beta$-actin was used to ensure equivalent loading of cell protein.

\section{TUNEL assay}

In situ cell apoptosis was determined with detection of fragmented DNA, using in situ cell death detection kit (Roche, Shanghai, China) according to the manufacturer's instructions.

\section{Tube formation assay}

96-well plates were coated with a thin layer of the Matrigel (BD Biosciences, CA, USA) and left to polymerize at $37^{\circ} \mathrm{C}$ for $0.5 \mathrm{~h}$. HUVEC $\left(2 \times 10^{4}\right.$ cells/well $)$ were starved overnight before being resuspended in $100 \mu \mathrm{L}$ endothelial cell medium and added to the polymerized Matrigel. After $8 \mathrm{~h}$ incubation, tube formation ability was assessed by calculating total tube length in three random fields using Wimasis Image Analysis Platform.

\section{Immunohistochemistry and immunofluorescence assay}

Immunohistochemistry was performed on $5 \mu \mathrm{m}$-paraffin sections with an indirect immunoperoxidase method 
using the primary antibody against FOXP3 (1:400, Cell Signaling, Beverly, MA, USA), CD31 (1:400, Abcam), pSTAT3 (1:400, Cell Signaling) and Bcl-2 (1:400, Cell Signaling). Microvessel density was scored semiquantitatively based on expression levels of CD31 positive cells, $+/++$ being some staining or rare vessel lumens, ++ $+/++++$ being some or more vessel lumens [20]. Immunofluorescence assay was performed on methanol-fixed cells or $5 \mu \mathrm{m}$-frozen sections using antibody against ICOS and ICOSL (Abcam). Texas red conjugated donkey anti-rabbit IgG antibody and FITC-conjugated goat anti-mouse IgG (Abcam) were used as the secondary antibody.

\section{Murine model}

To test the in vivo efficiency of ABT-199, BALB/c mice (56-week-old, obtained from Shanghai Laboratory Animal Center, Shanghai, China) were injected with $1 \times 10^{7}$ A20 cells into the right flank. Treatments started after tumor became about $0.5 \mathrm{~cm} \times 0.5 \mathrm{~cm}$ in surface (Day 0). For oral treatment, ABT-199 (10 mg/ml) was formulated in $60 \%$ phosal 50 propylene glycol, $30 \%$ polyethyleneglycol-400 and $10 \%$ ethanol. Tumor volumes were calculated as $0.5 \times$ $\mathrm{a}$ (length) $\times \mathrm{b}(\text { width })^{2}$.

\section{Transmission electron microscopy}

Ultrastructural studies focused on the morphology of tumor microvessels as previously described [19].

\section{Statistical analysis}

Difference of miR21 expression among groups was calculated using Mann-Whitney $U$ test. In vitro experimental results were expressed as mean \pm S.D. of data obtained from three separate experiments and determined by $t$-test to compare variance. Statistical significance was defined as $p<0.05$.

\section{Results}

Serum miR21 was elevated in B-cell lymphoma and indicated lymphoma progression

Comparing with healthy volunteers, serum miR21 was significantly increased in patients with DLBCL $(P=0.009$,
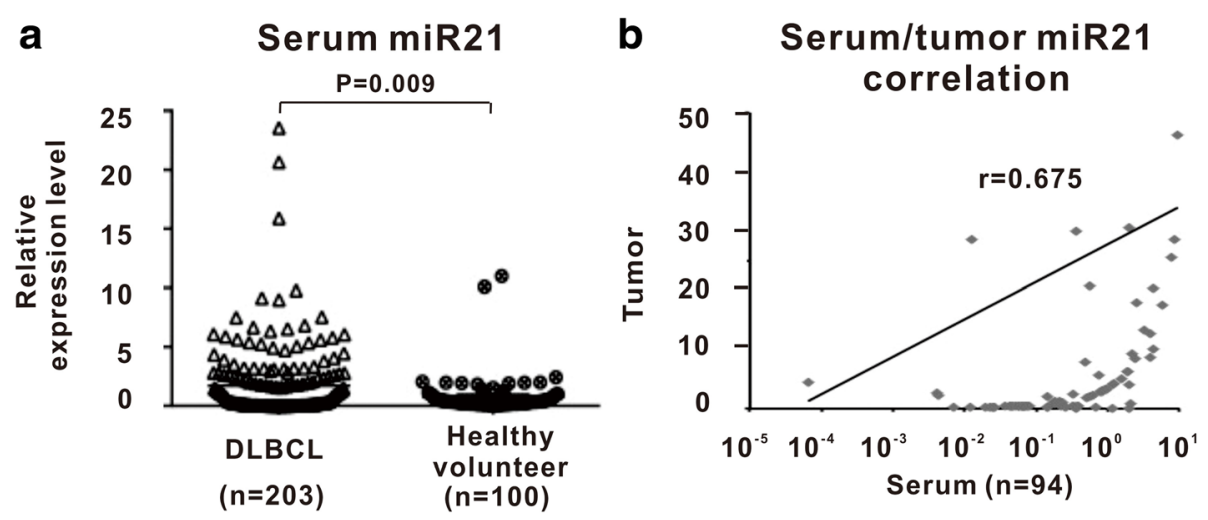

C

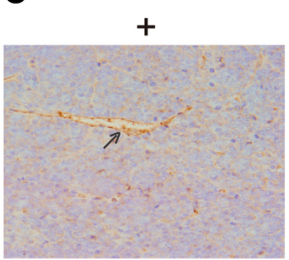

$+++$

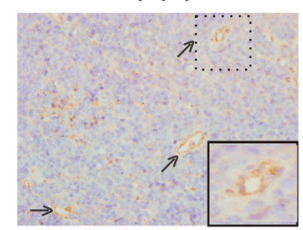

$++$

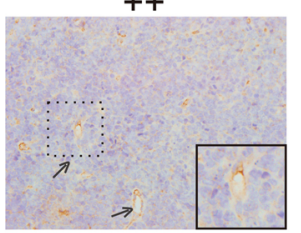

$+++$

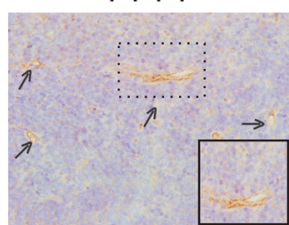

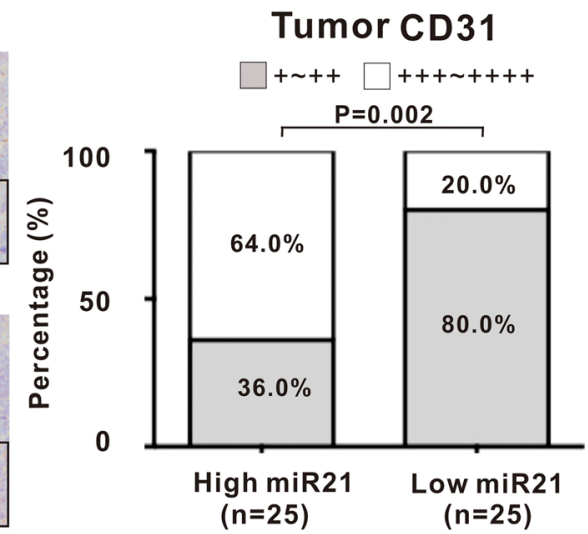

Fig. 1 Serum miR21 was elevated in B-cell lymphoma and indicated lymphoma progression. a Serum miR21 was significantly higher in DLBCL patients than in health volunteers. The relative expression level of each patient was calculated based on the lowest expression value. b A significant correlation was observed between serum and tumor miR21 expression level. Correlation coefficient was determined by Pearson correlation coefficient analysis. c Patients with high serum miR21 expression displayed significantly increased tumor CD31 positivity. The patients with miR21 expression level over and equal to the median value were regarded as high miR21 expression, while those below to the median value were included into the low miR21 expression 

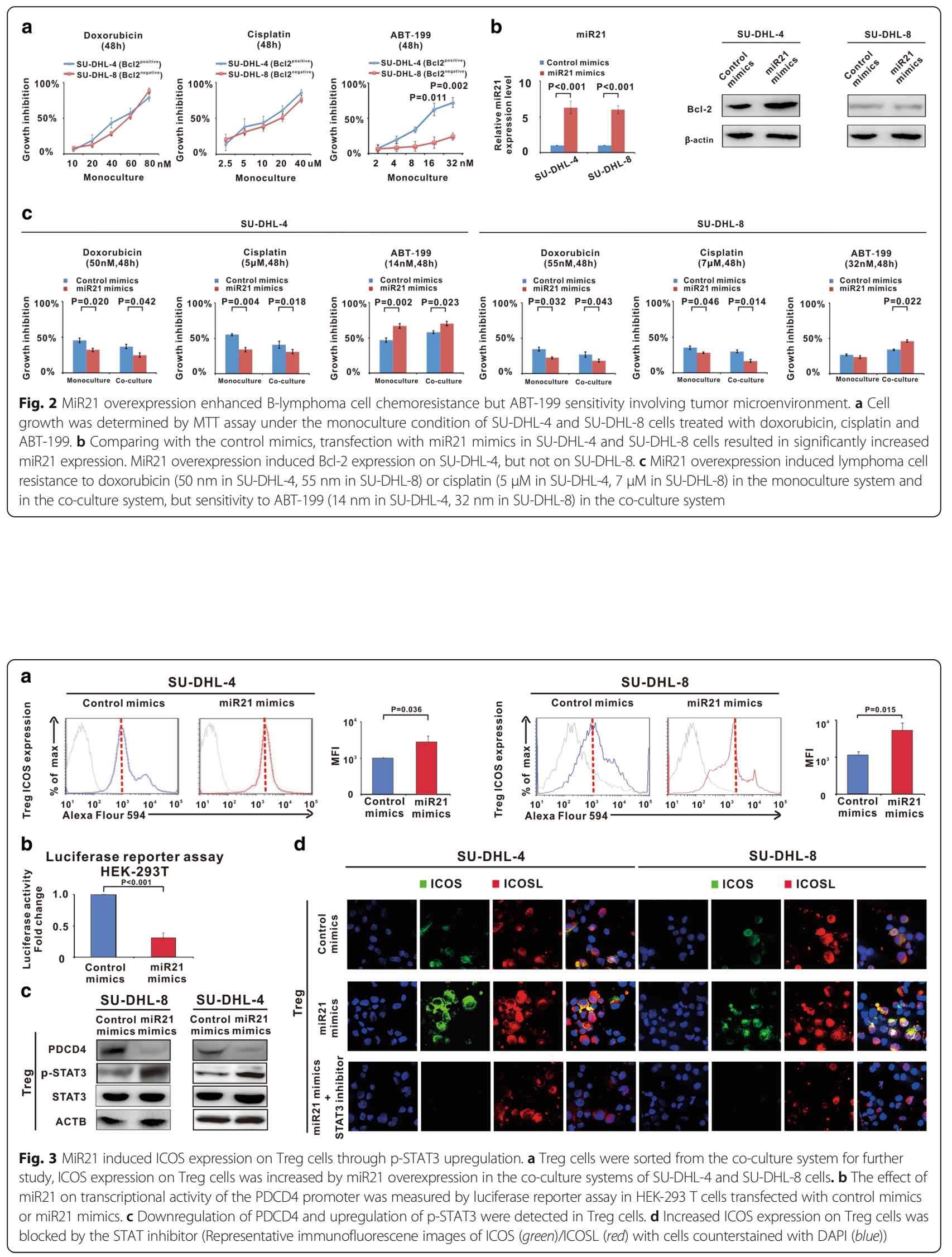
Fig. 1a). A significant correlation between serum and tumor miR21 expression was observed by Pearson correlation coefficient analysis $(r=0.675$, Fig. 1b). Elevated miR21 levels were associated with advanced Ann Arbor stage and International Prognostic Index indicating intermediate-high and high-risk $(P=0.027$ and $P=0.013$, Table 1). The median expression of miR21 was 0.318 in DLBCL. The patients with miR21 expression level over and equal to the median value were regarded as high miR21 expression, while those below the median value were included into low miR21 expression. As revealed by immunohistochemistry in tumor samples of DLBCL (25 each with high or low miR21 expression), CD31-positive microvessels were more frequently observed in high miR21 group than in low miR21 group $(P=0.002$, Fig. $1 \mathrm{c})$. These data suggested that serum miR21 was related to tumor progression and tumor angiogenesis in B-cell lymphoma.

\section{MiR21 overexpression enhanced B-lymphoma cell} chemoresistance but ABT-199 sensitivity involving tumor microenvironment

Bcl-2 $2^{\text {postive }}$ B-lymphoma cells SU-DHL- 4 and Bcl- $2^{\text {negative }}$ B-lymphoma cells SU-DHL-8 were treated with different concentrations of doxorubicin, cisplatin and ABT-199. Dose-response curves were shown in Fig. 2a. In the monoculture condition, compared with doxorubicin and cisplatin, the IC50 of ABT-199 in SU-DHL-8 was unachievable, confirming that the cytotoxic effect of ABT-199 relied on Bcl-2 expression in tumor cells. Accordingly, cell cycle arrest and cell apoptosis were observed in SU-DHL-4 cells, but not in SU-DHL-8 cells (Additional file 1: Figure S1A and B). To determine the biological function of miR21, SU-DHL-4 and SU-DHL-8 cells were transfected with miR21 mimics. Western blot showed that Bcl-2 protein level was increased in SU-DHL-4, while that of SU-DHL-8 was not inducible as previously reported [21] (Fig. 2b). While mimicking lymphoma microenvironment, cell proliferation assays were further performed in the co-culture system of lymphoma cells with immune cells and HUVEC cells. Different from the monoculture condition, miR21 overexpression resulted in lymphoma cell resistance to chemotherapeutic agents, but its sensitivity to ABT-199, is found not only in the co-culture system of $\mathrm{Bcl}-2^{\text {postive }}$ SU-DHL-4 cells, but also in the co-culture system of Bcl-2 ${ }^{\text {negative }}$ SU-DHL-8 cells (Fig. 2c). Therefore,
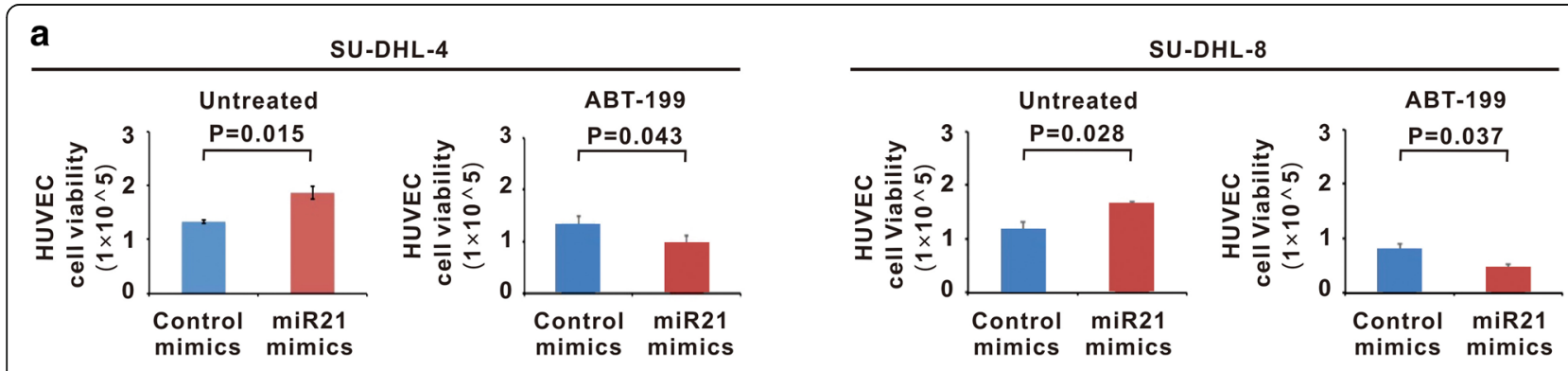

b

SU-DHL-4
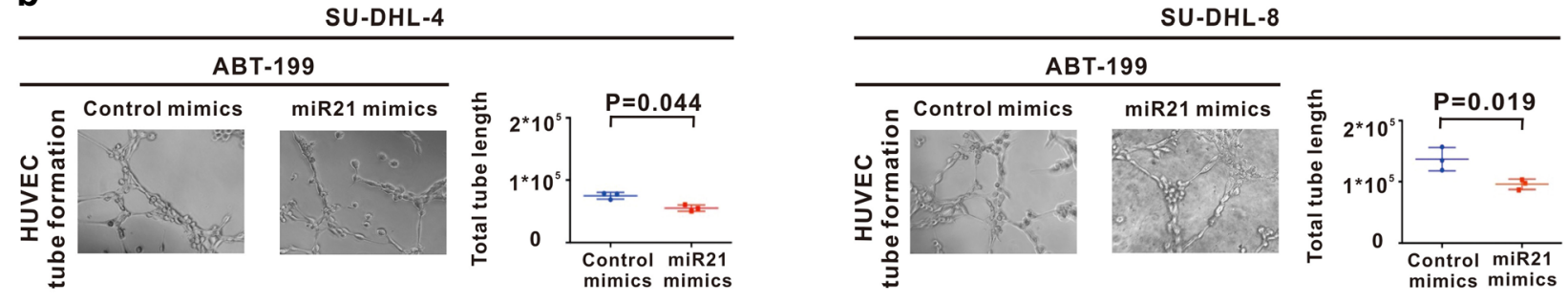

C

SU-DHL-4

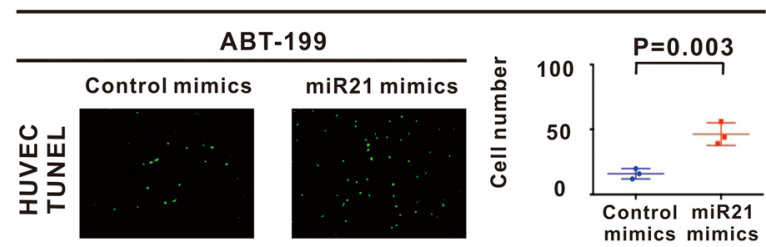

SU-DHL-8

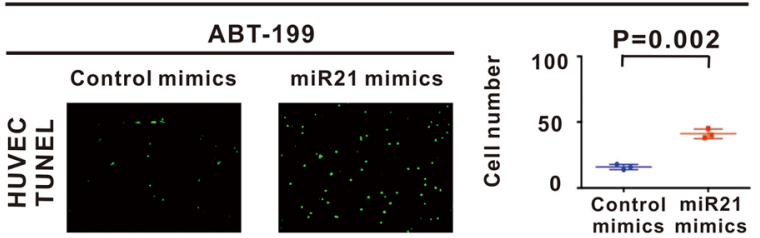

Fig. 4 ABT-199 counteracted miR21-mediated angiogenesis. a HUVEC cells were sorted from the co-culture system for further study, HUVEC growth was enhanced by miR21 overexpression and reduced by ABT-199 treatment in the co-culture systems of SU-DHL-4 and SU-DHL-8 cells. b-c ABT-199-mediated inhibition of HUVEC growth was associated with decreased tube formation (b) and increased HUVEC apoptosis, as revealed by TUNEL assay (c) 
irrespective of lymphoma cell Bcl-2 status, miR21 sensitized B-lymphoma cells to ABT-199 in the presence of tumor microenvironment.

\section{MiR21 induced ICOS expression on Treg cells through p-STAT3 upregulation}

To clarify the underlying mechanism behind miR21mediated sensitization of ABT-199 on B-cell lymphoma, Treg cells were sorted from the co-culture system and the effect of tumor miR21 on Treg cells was studied. Ectopic expression of miR21 of lymphoma cells significantly increased ICOS expression on Treg cells in both co-culture systems of SU-DHL-4 and SU-DHL-8 cells $(P=0.036$ and $P=0.015$, Fig. 3a). Previous study reported that miR21 regulates tumor progression through the miR21-PDCD4-STAT3 pathway [22]. As shown by luciferase reporter assay, miR21 repressed the transcriptional activity of the PDCD4 promoter (228$249 \mathrm{bp}$ ) in HEK-293 $\mathrm{T}$ cells (Fig. 3b). In Treg cells, PDCD4 was downregulated and phosphorylation of STAT3 was upregulated (Fig. 3c). Meanwhile, pharmacological inhibition of STAT3 by the STAT3 inhibitor abrogated the increased expression of ICOS on Treg cells induced by miR21 overexpression (Fig. 3d). Thus, miR21-mediated p-STAT3 phosphorylation was necessary for the induction of ICOS expression on Treg cells, independent of $\mathrm{Bcl}-2$ expression of lymphoma cells.

\section{ABT-199 counteracted miR21-mediated tumor angioge- nesis through ICOS/ICOSL-mediated interaction of Treg cells with endothelial cells}

HUVEC cells were also sorted from the co-culture system. In both co-culture systems of SU-DHL-4 and SUDHL-8 cells, ICOSL was stably expressed on HUVEC (Additional file 2: Figure S2). MiR21 overexpression of lymphoma cells significantly enhanced HUVEC growth ( $P=0.015$ and $P=0.028)$, which was retarded upon ABT-199 treatment $(P=0.043$ and $P=0.037$, Fig. 4a). Accordingly, vessel formation was reduced by ABT-199 $(P=0.044$ and $P=0.019$, Fig. $4 \mathrm{~b})$, in association with

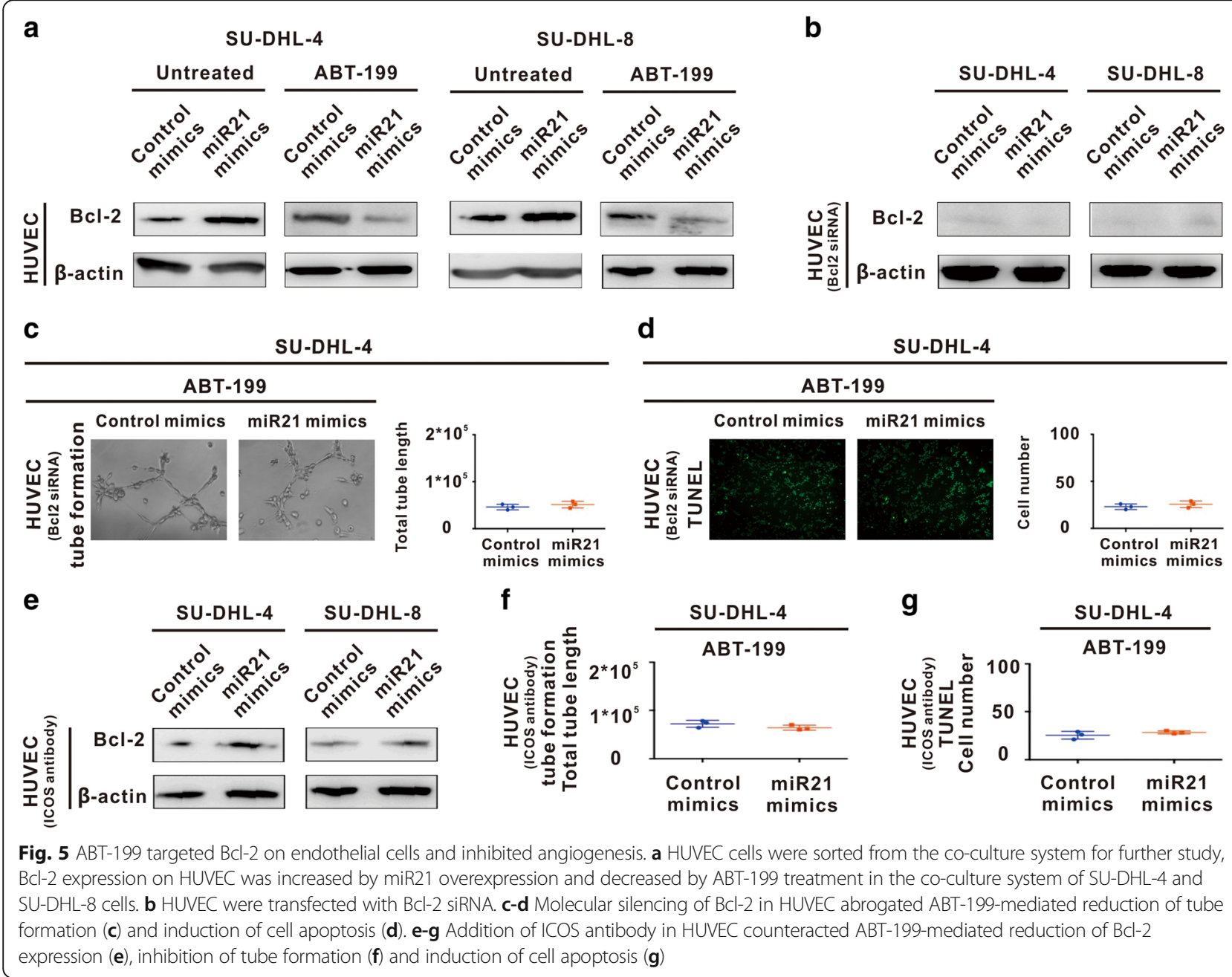


increased percentage of TUNEL positive endothelial cells $(P=0.003$ and $P=0.002$, Fig. $4 c)$.

Of note, Bcl-2 expression was increased on HUVEC co-cultured with miR21-overexpressing SU-DHL-4 cells or SU-DHL-8 cells, which was downregulated upon ABT-199 treatment (Fig. 5a). Bcl-2 was further knocked down by siRNA in HUVEC (Fig. 5b). As detected by tube formation assay and TUNEL assay, the effects of ABT-199 on HUVEC was abrogated by molecular silencing of Bcl-2 (Fig. 5c and d). Meanwhile, Bcl-2 expression remained constant when ICOS antibody was added to block the interaction between Treg cells and HUVEC (Fig. 5e). Similar results were obtained on tube formation and HUVEC apoptosis (Fig. $5 \mathrm{f}$ and $\mathrm{g}$ ).

Together, miR21 increased interaction of Treg cells with endothelial cells via ICOS/ICOSL axis and stimulated tumor angiogenesis, which was interrupted by ABT-199 through targeting Bcl-2 expression on endothelial cells.

\section{ABT-199 exhibited in vivo activity on} miR21-overexpressing lymphoma

Murine xenograft model was established with subcutaneous injection of A20 cells either stably transfected with pGMLV-miR21 or control vector pGMLV-ct. The tumor size of pGMLV-miR21 group was significantly larger than that of pGMLV-ct group $(P=0.043$ at Day 6 and $P=0.005$ at Day 7, Fig. 6a). ABT-199 treatment particularly exhibited anti-tumor activity on pGMLV-miR21 tumors, as compared to pGMLV-ct tumors $(P=0.045$ at Day 6 and $P=0.031$ at Day 7, Fig. 6a). Consistent with in vitro study, p-STAT3-positive Treg cells, as well as ICOS/ICOSL-mediated interaction of Treg cells with endothelial cells, were increased in untreated pGMLVmiR21 tumors (Fig. $6 \mathrm{~b}$ and c). Instead, Bcl-2-positive microvessels were reduced in pGMLV-miR21 tumors upon ABT-199 treatment (Fig. 6d), in parallel with the induction of apoptotic bodies in endothelial cells, as revealed by ultrastructural study (Fig. 6e).

\section{Discussion}

MiR21 is a key regulator of disease progression in B-cell lymphoma [23, 24]. Experimentally, the overexpression of miR21 leads to a pre-B malignant lymphoid-like phenotype [25]. In clinical settings, increased circulating miR21 level in sera from DLBCL patients is associated with matched tumor tissue, advanced disease stage and inferior overall survival [26, 27]. More recently, gene ontology and pathway analysis has suggested that cell-
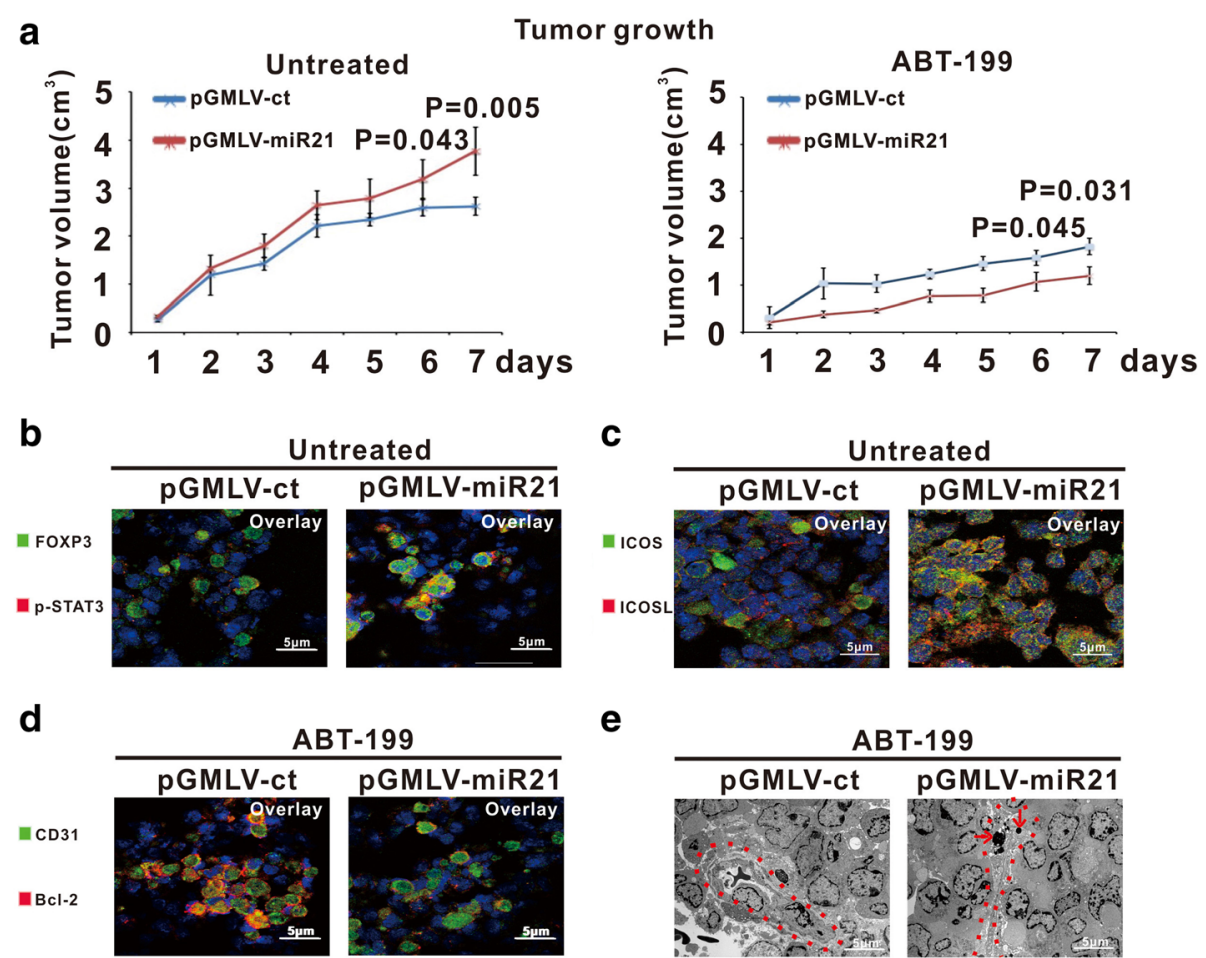

e

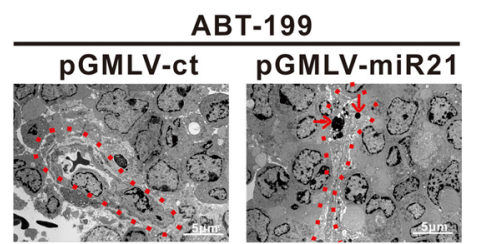

Fig. 6 ABT-199 exhibited in vivo activity on miR21-overexpressing lymphoma. a MiR21-overexpressing pGMLV-miR21 tumors grew more quickly than the control pGMLV-ct tumors and were more sensitive to ABT-199 treatment. b-c p-STAT3-positive Treg cells (b) and ICOS/ICOSL interaction (c) were decreased in untreated pGMLV-miR21 tumors. $\mathbf{d}$-e Bcl-2 expression (d) and cell apoptosis revealed by ultrastructural analysis (e) were increased in microvessels of ABT-199-treated pGMLV-miR21 tumors. Microvessels were indicated by red broken lines and apoptotic bodies by red arrow 
environment interaction is another important miR21 pathogenic mechanism [28]. Here we not only confirmed miR21 as a serum oncogenic biomarker of DLBCL, but also provided a direct link of miR21 with lymphoma progression and tumor angiogenesis.

Dysregulated tumor microenvironment determines cancer cell chemosensitivity [29]. Our study showed that ectopic expression of miR21 led to chemoresistance of Blymphoma cells, more profoundly when lymphoma cells were co-cultured with immune cells and endothelial cells, main components of tumor microenvironment. This is in accordance with previous studies which showed miR21 provokes myeloma cell adhesion to bone marrow stromal cells and resistance to chemotherapeutic agents [30], in addition, miR21 initiates inflammatory signaling in HER2positive breast cancer and reduces the cytotoxic effect of neoadjuvant trastuzumab and chemotherapy [31].

Crosstalk between Treg cells and endothelial cells is essential in drug resistance [6]. ICOS, being a member of the CD28 family of co-stimulatory molecules, is implicated in maintaining durable immune reactions upon binding to ICOSL [32, 33]. Particularly, ICOS/ICOSL axis plays a pivotal role in Treg cell function and promotes Treg differentiation through activating the phosphoinositide 3-kinaseAkt pathway [34, 35]. MiR21 can modulate ICOS-ICOSL expression and contribute to the progression of colorectal cancer [36]. To our knowledge, we provided the first evidence that miR21 enhanced the interaction of Treg cells with endothelial cells, induced ICOS expression on Treg cells, stimulated tumor angiogenesis via ICOS/ICOSL signaling, and led to chemoresistance of B-cell lymphoma. Recent study showed that follicular lymphoma cells generate Treg cells via ICOS/ICOSL cascade and are susceptible to anti-ICOS/ICOSL therapy [37]. Therefore, our results highlighted a key role for Treg cells in DLBCL progression and suggested that targeting ICOS/ICOSL pathway may be an alternative immunotherapy for DLBCL treatment.

ABT-199 can specially target Bcl-2 on tumor cells and induces B-lymphoma cell apoptosis. However, it is unclear whether ABT-199 would affect tumor microenvironment in a $\mathrm{Bcl}$-2-dependent manner. Here, both in vitro and in vivo, our study characterized a $\mathrm{Bcl}$-2-dependent inhibition of ABT-199 on tumor angiogenesis, mediated by intrinsic miR21-Treg cell pathway, leading to sensitizing effect of ABT-199 on B-cell lymphoma. Therefore, in addition to the oncogenic role of miR21 on malignant B-lymphocytes [38], miR21 possessed a potential activity on tumor microenvironment, indicative an alternative mechanism of Blymphoma cell response to ABT-199.

The above data demonstrated that miR21 plays an oncogenic role in B-cell lymphoma by modulating tumor microenvironment and supported clinical rationale for using miR21 as a biomarker to select chemoresistant B-lymphoma patients who may benefit from ABT-199 treatment.

\section{Additional files}

Additional file 1: Figure S1. Cell apoptosis and cell cycle of SU-DHL-4 and SU-DHL-8 cells before and after ABT-199 treatment. A: ABT-199 induced cell apoptosis in SU-DHL-4, but not in SU-DHL-8 cells. B: No cell cycle arrest was observed in SU-DHL-4 and SU-DHL-8 upon ABT-199 treatment. (JPG $247 \mathrm{~kb}$ )

Additional file 2: Figure S2. ICOSL expression on HUVEC cells in the co-culture systems of SU-DHL-4 and SU-DHL-8 cells. ICOSL was stably expressed on HUVEC cells in the co-culture systems of SU-DHL-4 and SU-DHL-8 cells. (JPG 203 kb)

\section{Abbreviations}

DLBCL: Diffuse large B-cell lymphoma; HUVEC: Human umbilical vein endothelial cell; ICOS: Inducible co-stimulator; ICOSL: ICOS ligand; IPI: International prognostic index; LDH: Lactate dehydrogenase; MiRs: MicroRNAs; Treg: Regulatory T cells

\section{Acknowledgement}

We appreciate the effort the physicians for enrolling patients and thank all the patients involved for allowing us to analyze their clinical data.

\section{Funding}

This study was supported, in part, by research funding from the National Natural Science Foundation of China (81325003, 81520108003, 81670716 and 81201863), the National Key Research and Development Program (2016YFC0902800), the Shanghai Commission of Science and Technology (14430723400, 14140903100 and 16JC1405800), the Shanghai Municipal Education Commission Gaofeng Clinical Medicine Grant Support (20152206 and 20152208), Multi-center clinical research project by Shanghai Jiao Tong University School of Medicine (DLY201601), Chang Jiang Scholars Program, Collaborative Innovation Center of Systems Biomedicine and the Samuel Waxman Cancer Research Foundation.

\section{Availability of data and materials}

ZZ, PPX, LW and HJZ had full access to all the data in the study (available upon specific data request). Although our data is de-identified, we determine not to share these in public due to further study on this subject. However, we would like to share the data to other researchers if necessary. All of the methods or reagents we used are accessible on the market.

\section{Authors' contributions}

ZZ, LW, HJZ and JX performed experiments; PPX, HJZ and YZ analyzed clinical data; XQW, BQ and XFW gave technical support; WLZ and AJ designed the study, directed and supervised the research and wrote the manuscript.

\section{Competing interest}

The authors declare no conflict of interest.

Consent for publication

Not applicable.

\section{Ethics approval and consent to participate}

The study was approved by Shanghai Rui Jin Hospital review board with informed consent obtained in accordance with the Declaration of Helsinki. Animals were used according to the protocols approved by Shanghai Rui Jin Hospital Animal Care and Use Committee.

\section{Publisher's Note}

Springer Nature remains neutral with regard to jurisdictional claims in published maps and institutional affiliations.

\section{Author details}

${ }^{1}$ State Key Laboratory of Medical Genomics, Shanghai Institute of Hematology, Shanghai Rui Jin Hospital, Shanghai Jiao Tong University School of Medicine, 197 Rui Jin Er Road, Shanghai 200025, China. ${ }^{2}$ Pôle de Recherches Sino-Français en Science du Vivant et Génomique, Laboratory of Molecular Pathology, Shanghai, China. ${ }^{3}$ Department of Laboratory Medicine, 
Shanghai Rui Jin Hospital, Shanghai Jiao Tong University School of Medicine, Shanghai, China. ${ }^{4}$ U1165 Inserm/Université Paris 7, Hôpital Saint Louis, Paris, France.

Received: 13 March 2017 Accepted: 13 June 2017

Published online: 21 June 2017

\section{References}

1. Sehn LH, Gascoyne RD. Diffuse large B-cell lymphoma: optimizing outcome in the context of clinical and biologic heterogeneity. Blood. 2015;125(1):22-32.

2. Souers AJ, Leverson JD, Boghaert ER, Ackler SL, Catron ND, Chen J, Dayton BD, Ding H, Enschede SH, Fairbrother WJ, et al. ABT-199, a potent and selective BCL-2 inhibitor, achieves antitumor activity while sparing platelets. Nat Med. 2013;19(2):202-8

3. Vandenberg CJ, Cory S. ABT-199, a new BCl-2-specific BH3 mimetic, has in vivo efficacy against aggressive Myc-driven mouse lymphomas without provoking thrombocytopenia. Blood. 2013;121(12):2285-8.

4. Konopleva MY, Jordan CT. Leukemia stem cells and microenvironment: biology and therapeutic targeting. J Clin Oncol. 2011;29(5):591-9.

5. Carmeliet $P$, Jain RK. Principles and mechanisms of vessel normalization for cancer and other angiogenic diseases. Nat Rev Drug Discov. 2011;10(6):417-27.

6. Motz GT, Coukos $\mathrm{G}$. The parallel lives of angiogenesis and immunosuppression: cancer and other tales. Nat Rev Immunol. 2011;11(10):702-11.

7. Shain KH, Dalton WS, Tao J. The tumor microenvironment shapes hallmarks of mature B-cell malignancies. Oncogene. 2015;34(36):4673-82.

8. Curiel TJ. Tregs and rethinking cancer immunotherapy. J Clin Invest. 2007;117(5):1167-74

9. Scott DW, Gascoyne RD. The tumour microenvironment in B cell lymphomas. Nat Rev Cancer. 2014;14(8):517-34.

10. Dianzani C, Minelli R, Mesturini R, Chiocchetti A, Barrera G, Boscolo S, Sarasso C, Gigliotti CL, Sblattero D, Yagi J, et al. B7h triggering inhibits umbilical vascular endothelial cell adhesiveness to tumor cell lines and polymorphonuclear cells. J Immunol. 2010;185(7):3970-9.

11. Chiron D, Dousset C, Brosseau C, Touzeau C, Maiga S, Moreau P, Pellat-Deceunynck C, Le Gouill S, Amiot M. Biological rational for sequential targeting of Bruton tyrosine kinase and $\mathrm{BCl}-2$ to overcome CD40-induced ABT-199 resistance in mantle cell lymphoma. Oncotarget. 2015;6(11):8750-9.

12. Lee $D$, Shin C. MicroRNA-target interactions: new insights from genome-wide approaches. Ann N Y Acad Sci. 2012;1271:118-128.

13. Kim JW, Mori S, Nevins JR. Myc-induced microRNAs integrate Myc-mediated cell proliferation and cell fate. Cancer Res. 2010;70(12):4820-8.

14. Cheng C, Bahal R, Babar IA, Pincus Z, Barrera F, Liu C, Svoronos A, Braddock DT, Glazer PM, Engelman DM, et al. MicroRNA silencing for cancer therapy targeted to the tumour microenvironment. Nature. 2015;518(7537):107-10.

15. Dong J, Zhao YP, Zhou L, Zhang TP, Chen G. Bcl-2 upregulation induced by miR-21 via a direct interaction is associated with apoptosis and chemoresistance in MIA PaCa-2 pancreatic cancer cells. Arch Med Res. 2011;42(1):8-14.

16. Fang C, Zhu DX, Dong HJ, Zhou ZJ, Wang YH, Liu L, Fan L, Miao KR, Liu P, Xu W, et al. Serum microRNAs are promising novel biomarkers for diffuse large B cell lymphoma. Ann Hematol. 2012;91(4):553-9.

17. Lawrie CH, Gal S, Dunlop HM, Pushkaran B, Liggins AP, Pulford K, Banham AH, Pezzella F, Boultwood J, Wainscoat JS, et al. Detection of elevated levels of tumour-associated microRNAs in serum of patients with diffuse large B-cell lymphoma. Br J Haematol. 2008;141(5):672-5.

18. Zheng Z, Cheng S, Wu W, Wang L, Zhao Y, Shen Y, Janin A, Zhao WL. C-FLIP is involved in tumor progression of peripheral T-cell lymphoma and targeted by histone deacetylase inhibitors. J Hematol Oncol. 2014;7:88.

19. Dong LH, Cheng S, Zheng Z, Wang L, Shen Y, Shen ZX, Chen SJ, Zhao WL. Histone deacetylase inhibitor potentiated the ability of MTOR inhibitor to induce autophagic cell death in Burkitt leukemia/lymphoma. J Hematol Oncol. 2013;6:53.

20. Berger AC, Feldman AL, Gnant MF, Kruger EA, Sim BK, Hewitt S, Figg WD, Alexander HR, Libutti SK. The angiogenesis inhibitor, endostatin, does not affect murine cutaneous wound healing. J Surg Res. 2000;91(1):26-31.

21. Deng J, Carlson N, Takeyama K, Dal Cin P, Shipp M, Letai A. BH3 profiling identifies three distinct classes of apoptotic blocks to predict response to ABT-737 and conventional chemotherapeutic agents. Cancer Cell. 2007; 12(2):171-85.

22. Jiang LH, Ge MH, Hou XX, Cao J, Hu SS, Lu XX, Han J, Wu YC, Liu X, Zhu X, et al. miR-21 regulates tumor progression through the miR-21-PDCD4-Stat3 pathway in human salivary adenoid cystic carcinoma. Lab Invest. 2015;95(12):1398-408.

23. Lawrie $\mathrm{CH}$. MicroRNAs and lymphomagenesis: a functional review. $\mathrm{Br} J$ Haematol. 2013;160(5):571-81.

24. Baraniskin A, Kuhnhenn J, Schlegel U, Chan A, Deckert M, Gold R, Maghnouj A, Zollner $H$, Reinacher-Schick A, Schmiegel W, et al. Identification of microRNAs in the cerebrospinal fluid as marker for primary diffuse large B-cell lymphoma of the central nervous system. Blood. 2011;117(11):3140-6.

25. Medina PP, Nolde M, Slack FJ. OncomiR addiction in an in vivo model of microRNA-21-induced pre-B-cell lymphoma. Nature. 2010;467(7311):86-90.

26. Li J, Fu R, Yang L, Tu W. miR-21 expression predicts prognosis in diffuse large B-cell lymphoma. Int J Clin Exp Pathol. 2015;8(11):15019-24.

27. Chen W, Wang H, Chen H, Liu S, Lu H, Kong D, Huang X, Kong Q, Lu Z. Clinical significance and detection of microRNA-21 in serum of patients with diffuse large B-cell lymphoma in Chinese population. Eur J Haematol. 2014;92(5):407-12

28. Chen B, Chen X, Wu X, Wang X, Wang Y, Lin TY, Kurata J, Wu J, Vonderfecht S, Sun $G$, et al. Disruption of microRNA-21 by TALEN leads to diminished cell transformation and increased expression of cell-environment interaction genes. Cancer Lett. 2015;356(2 Pt B):506-16.

29. An G, Acharya C, Feng X, Wen K, Zhong M, Zhang L, Munshi NC, Qiu L, Tai YT, Anderson KC. Osteoclasts promote immune suppressive microenvironment in multiple myeloma: therapeutic implication. Blood. 2016;128(12):1590-603.

30. Wang X, Li C, Ju S, Wang Y, Wang H, Zhong R. Myeloma cell adhesion to bone marrow stromal cells confers drug resistance by microRNA-21 up-regulation. Leuk Lymphoma. 2011:52(10):1991-8.

31. De Mattos-Arruda L, Bottai G, Nuciforo PG, Di Tommaso L, Giovannetti E, Peg V, Losurdo A, Perez-Garcia J, Masci G, Corsi F, et al. MicroRNA-21 links epithelial-to-mesenchymal transition and inflammatory signals to confer resistance to neoadjuvant trastuzumab and chemotherapy in HER2-positive breast cancer patients. Oncotarget. 2015;6(35):37269-80.

32. Sharpe AH, Freeman GJ. The B7-CD28 superfamily. Nat Rev Immunol. 2002:2(2):116-26.

33. Ito T, Hanabuchi S, Wang YH, Park WR, Arima K, Bover L, Qin FX, Gilliet M, Liu YJ. Two functional subsets of FOXP3+ regulatory $T$ cells in human thymus and periphery. Immunity. 2008;28(6):870-80.

34. Lee HJ, Kim SN, Jeon MS, Yi T, Song SU. ICOSL expression in human bone marrow-derived mesenchymal stem cells promotes induction of regulatory T cells. Sci Rep. 2017:7:44486.

35. Busse M, Krech M, Meyer-Bahlburg A, Hennig C, Hansen G. ICOS mediates the generation and function of $\mathrm{CD} 4+\mathrm{CD} 25+$ Foxp3 + regulatory $T$ cells conveying respiratory tolerance. J Immunol. 2012;189(4):1975-82.

36. Wu D, Tang R, Qi Q, Zhou X, Zhou H, Mao Y, Li R, Liu C, Wang W, Hua D, et al. Five functional polymorphisms of B7/CD28 co-signaling molecules alter susceptibility to colorectal cancer. Cell Immunol. 2015;293(1):41-8.

37. Le KS, Thibult ML, Just-Landi S, Pastor S, Gondois-Rey F, Granjeaud S, Broussais F, Bouabdallah R, Colisson R, Caux C, et al. Follicular B lymphomas generate regulatory $T$ cells via the ICOS/ICOSL pathway and Are susceptible to treatment by anti-ICOS/ICOSL therapy. Cancer Res. 2016;76(16):4648-60.

38. Go H, Jang JY, Kim PJ, Kim YG, Nam SJ, Paik JH, Kim TM, Heo DS, Kim CW, Jeon YK. MicroRNA-21 plays an oncogenic role by targeting FOXO1 and activating the PI3K/AKT pathway in diffuse large B-cell lymphoma. Oncotarget. 2015;6(17):15035-49.

\section{Submit your next manuscript to BioMed Central and we will help you at every step:}

- We accept pre-submission inquiries

- Our selector tool helps you to find the most relevant journal

- We provide round the clock customer support

- Convenient online submission

- Thorough peer review

- Inclusion in PubMed and all major indexing services

- Maximum visibility for your research

Submit your manuscript at www.biomedcentral.com/submit 